Journal of the Electrochemical Society, Vol. 146, No. 11, 1999, pp. 4095-4100.

ISSN: 0013-4651

DOI: $10.1149 / 1.1392597$

http://www.electrochem.org/

http://scitation.aip.org/getpdf/servlet/GetPDFServlet?filetype=pdf\&id=JESOAN000146000011004095000001\&idty pe $=$ cvips\&prog $=$ normal

(C) The Electrochemical Society, Inc. 1999. All rights reserved. Except as provided under U.S. copyright law, this work may not be reproduced, resold, distributed, or modified without the express permission of The Electrochemical Society (ECS). The archival version of this work was published in Journal of the Electrochemical Society, Vol. 146, No. 11, 1999, pp. 4095-4100.

\title{
The Influence of Dichromate Ions on Aluminum Dissolution Kinetics in Artificial Crevice Electrode Cells
}

\author{
E. Akiyama and G. S. Frankel* \\ Fontana Corrosion Center, The Ohio State University, Columbus, Ohio \\ *Electrochemical Society Active Member.
}

Dissolution kinetics for pits and crevices in aluminum and the effect of dichromate ions on the dissolution kinetics were investigated by using artificial crevice electrodes. The aluminum artificial crevice electrodes were potentiostatically polarized over a range of potential in $0.1 \mathrm{M} \mathrm{NaCl}$ solution with and without dichromate ions. The anodic dissolution charge, and cathodic charges for the hydrogen and dichromate reduction reactions, were measured. The addition of dichromate ions did not suppress the active dissolution. This indicates that the mechanism of localized corrosion inhibition by dichromates is something other than anodic inhibition of Al dissolution in the pit or crevice environment. The relative amount of local cathodic reactions on $\mathrm{Al}$ was increased by the addition of dichromate because of the dichromate reduction. The initial dissolution of aluminum in a crevice was ohmic controlled. From the change in the dissolution current with time, the conductivity of the crevice and potential at the bottom of crevice were estimated. The conductivity and the bottom potential decreased with the ratio of cathodic charge of hydrogen evolution to anodic dissolution charge. The conductivity in the crevice and thus the dissolution current seem to be controlled by hydrogen evolution and only indirectly by dichromate concentration.

The influence of chromate on pitting of aluminum and aluminum alloys is well-known, and chromate-based corrosion inhibitors and coatings are widely used for the protection of $\mathrm{Al}$ and its alloys. However, the mechanism of the inhibition effect of chromates is still unclear. One possible explanation for the inhibition of localized corrosion of aluminum by chromates is that they reduce the local dissolution kinetics, effectively preventing pitting from occurring.

In order to study various aspects of the kinetics of pit growth, artificial pit or crevice electrodes have been used by researchers previously. ${ }^{1-7}$ The most common geometry is the "leadin-pencil" electrode consisting of a thin metal wire in an insulating mounting. The dissolution of the thin wire forms a cylindrical pit in which only the pit bottom is active, so that the active metal area is known. Also, artificial pit electrodes develop a local environment identical to real pits and crevices. Therefore, the electrode provides information on the dissolution kinetics in localized corrosion regions such as pits and crevices. Furthermore, mass transfer is parallel to the in-depth axis so these electrodes provide a simplified one-dimensional transport situation.

Isaacs reported that uniform diffusion-controlled dissolution was established when an artificial pit electrode of stainless steel was polarized in $1 \mathrm{M} \mathrm{NaCl}$ solution above about $200 \mathrm{mV}$ (SCE). ${ }^{1,3}$ The diffusion-controlled current indicated that the metal surface is covered by a salt film with resistive impedance up to high frequencies. ${ }^{3}$ Similar salt film properties were reported by Beck for iron. ${ }^{5}$ Ferrous salt films formed in hydrochloric, perchloric, and sulfuric acid 
solutions at potentials and current densities above threshold values. Below the passivation potential, a salt film remains, and a limiting current density for iron dissolution was determined by mass-transport conditions.

Heimgartner and Böhni measured the current/time behavior for $\mathrm{Ni}$ artificial pits in dilute chloride solutions and reported a change in dissolution mechanism from ohmic-charge transfer to diffusion control. ${ }^{7}$ The following parameters were found to increase the transition time for the mechanism change: low potentials, positioning of the electrode that allowed the concentrated corrosion products to flow out of the pit, agitation of the bulk electrolyte, and large pit diameters.

The effect of hydrodynamics on pitting rate was investigated quantitatively by Beck using an artificial pit with $0.32 \times 0.32 \mathrm{~cm}$ square cross section in the side of a flow channel for iron, titanium and aluminum in chloride, bromide, and iodide solutions. ${ }^{8}$ He reported that two modes of pitting were observed: high-current density pits with growth limited only by ohmic resistance in solution, and lower-current density limited by mass transfer. Only the ohmic mode was observed for aluminum and for titanium in chloride solution. Corrosion in these cases was accompanied by a large evolution of hydrogen, which causes local agitation of the diffusion even at a zero solution flow. Mass-transfer limited dissolution was observed for iron in concentrated chloride and titanium in bromide and iodide at low solution flow rate. Beck also reported that a combination of concentrated aluminum chloride solution ( $>80 \%$ of saturation) and high anodic potentials resulted in the formation of continuous salt film and electropolishing of the surface of pure Al rods with $5 \mathrm{~mm}^{2}$ cross section imbedded in epoxy resin. ${ }^{4}$ Current density under these conditions was mass-transfer controlled and increased with solution flow. At low salt concentrations and potentials, the convection induced by simultaneous hydrogen evolution prevented continuous salt film formation.

The artificial crevice electrode geometry is similar to that of the artificial pit, and is formed by sandwiching a foil electrode between two insulating sheets. ${ }^{9}$ Dissolution of the foil results in a slot-like crevice. Artificial crevice electrodes allow for easy visual observation of the dissolving surface. Furthermore, when the insulating sidewall is thin, there is easy access to the pit environment for analytical probes. The depth distribution and valence of ion species in artificial crevices have been investigated by Isaacs et al. using X-ray fluorescence techniques. ${ }^{9-11}$ For instance, an in situ study of anodic dissolution of stainless steel in chloride solution found that the salt layer formed on nickel/chromium steels was rich in iron and contained little nickel and a very small amount of chromium. ${ }^{9}$

In an attempt to understand the dissolution kinetics for pits and crevices in aluminum and to determine if dichromate inhibits localized corrosion by reducing the dissolution kinetics, the electrochemical behavior of aluminum artificial crevice electrodes in chloride-containing solutions with and without dichromate ions was investigated in the present work.

\section{Experimental}

Figure 1 shows a schematic drawing of the electrochemical cell used. The structure of the artificial crevice cell is similar to that previously used by other researchers such as Isaacs and Newman for ferrous metals and alloys. ${ }^{1,9,12,13}$ A piece of Al 1100 foil (Al 99.23, Fe 0.50, Cu 0.15, Si 0.11, Zn 0.006, Mn 0.004, Mg<0.001 wt \%) with $50.8 \mu \mathrm{m}$ thickness and about $6.7 \mathrm{~mm}$ width was sandwiched between two transparent sheets using epoxy resin. This electrode provides a nearly one-dimensional diffusion geometry and allows for easy visual observation.

The sandwich of $\mathrm{Al}$ foil/transparent sheets was attached to a Plexiglas cell that consisted 
of a $1.5 \times 1.5 \mathrm{~cm}$ square tube attached

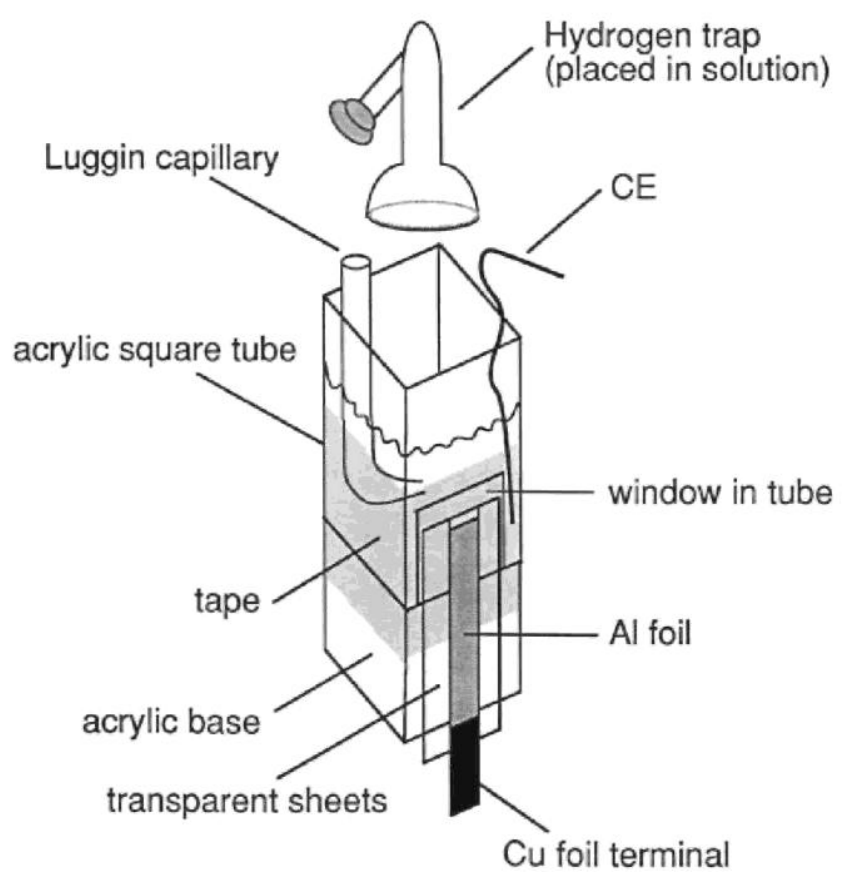

Figure 1. A schematic drawing of the artificial crevice cell.

to a solid base. The top of the sandwich was positioned at a window in the side of the cell, and the window was sealed with polyester (Mylar) tape. Paraffin tape was wrapped around the cell to avoid leaks. A platinum wire and a saturated calomel electrode (SCE) were used for the counter and reference electrodes, respectively. All potentials are referenced to the SCE.

In order to collect hydrogen gas evolved simultaneously from the Al electrodes during Al dissolution, a gas trap shown in Fig. 1 was placed in the solution over the metal electrode. The inside of the gas trap was filled with solution by evacuating the air in the trap using a syringe. The volume of hydrogen gas collected was measured after the experiment using a microsyringe.

The aluminum artificial crevice electrodes were potentiostatically polarized over a range of potential from $0-1 \mathrm{~V}(\mathrm{SCE})$ in $0.1 \mathrm{M} \mathrm{NaCl}$ solution with varying amounts of $\mathrm{Na}_{2} \mathrm{Cr}_{2} \mathrm{O}_{7}(0$, 0.01 , or $0.1 \mathrm{M}$ ) for $2 \mathrm{~h}$ at room temperature. The current density and net charge, $Q_{\text {net, }}$, were recorded from the EG\&G PAR 273 potentiostat. Before each measurement, the top of the thin foil electrode was cut in order to expose a fresh surface (with no recess) to the solution at the start of the experiment.

$Q_{\text {ano, }}$, the charge associated with $\mathrm{Al}$ dissolution at the active crevice surface, was measured by image analysis of the electrodes at the end of the experiment after disassembly of the cell. The image of the dissolved electrode was scanned and magnified, and the dissolved area and thus the volume dissolved were measured. The anodic charge was determined from Faraday's law 


$$
Q_{\mathrm{ano}}=A d \rho n F / M
$$

where $A\left(\mathrm{~cm}^{2}\right)$ is dissolved area, $d\left(5.08 \times 10^{-3} \mathrm{~cm}\right)$ is thickness of the Al foil, $\rho$ is the density of $\mathrm{Al}\left(2.7 \mathrm{~g} / \mathrm{cm}^{3}\right), n$ for $\mathrm{Al}$ is 3 equiv/mol, $F(96,487 \mathrm{C} /$ equiv) is Faraday's constant, and $M$ is atomic mass of $\mathrm{Al}(27 \mathrm{~g} / \mathrm{mol})$.

The net charge passing through the potentiostat, $Q_{\text {net }}$, is the difference between the total anodic charge, $Q_{\mathrm{ano}}$, and the (absolute value of the) charge of the local cathodic reaction on the Al electrode, $Q_{\text {cat }}$, because significant cathodic reactions take place on the Al electrodes. The primary cathodic reactions on the $\mathrm{Al}$ electrode are hydrogen evolution and dichromate reduction

$$
Q_{\text {net }}=Q_{\text {ano }}-Q_{\text {cat }}=Q_{\text {ano }}-\left(Q_{\mathrm{H}_{2}}+Q_{\mathrm{Cr}} 6+\right)
$$

where $Q_{\mathrm{H} 2}$ is the charge of hydrogen evolution on the $\mathrm{Al}$ electrode, and $Q_{\mathrm{Cr}} 6+$ is that of dichromate reduction. The charge associated with oxygen reduction is assumed to be small in comparison. $Q_{\mathrm{H} 2}$ was determined from the volume of hydrogen gas collected, as described above. This assumes a collection efficiency of $100 \%$, which is an overestimate since some of the evolved hydrogen gas will dissolve into solution or remain as bubbles in the crevice. Nonetheless, $Q_{\mathrm{Cr}} 6+$ was calculated from Eq. 2, given the measured values of $Q_{\text {net }}, Q_{\mathrm{ano}}$, and $Q_{\mathrm{H} 2}$.

The conductivities of bulk solutions were measured with a YSI 3200 conductivity meter.

\section{Results and Discussion}

Figure 2 shows the effect of applied potential on net charge, $Q_{\text {net }}$, anodic dissolution charge, $Q_{\text {ano, }}$ and the charge of local cathodic reaction on $\mathrm{Al}, Q_{\text {cat }}$, for the $2 \mathrm{~h}$ potentiostatic polarization in the three chloride solutions containing different concentrations of dichromate. The addition of dichromate to the solution does not significantly decrease the anodic dissolution charge during crevice growth. Accordingly, the mechanism of localized corrosion inhibition by chromates must be something other than anodic inhibition in the pit or crevice environment. 

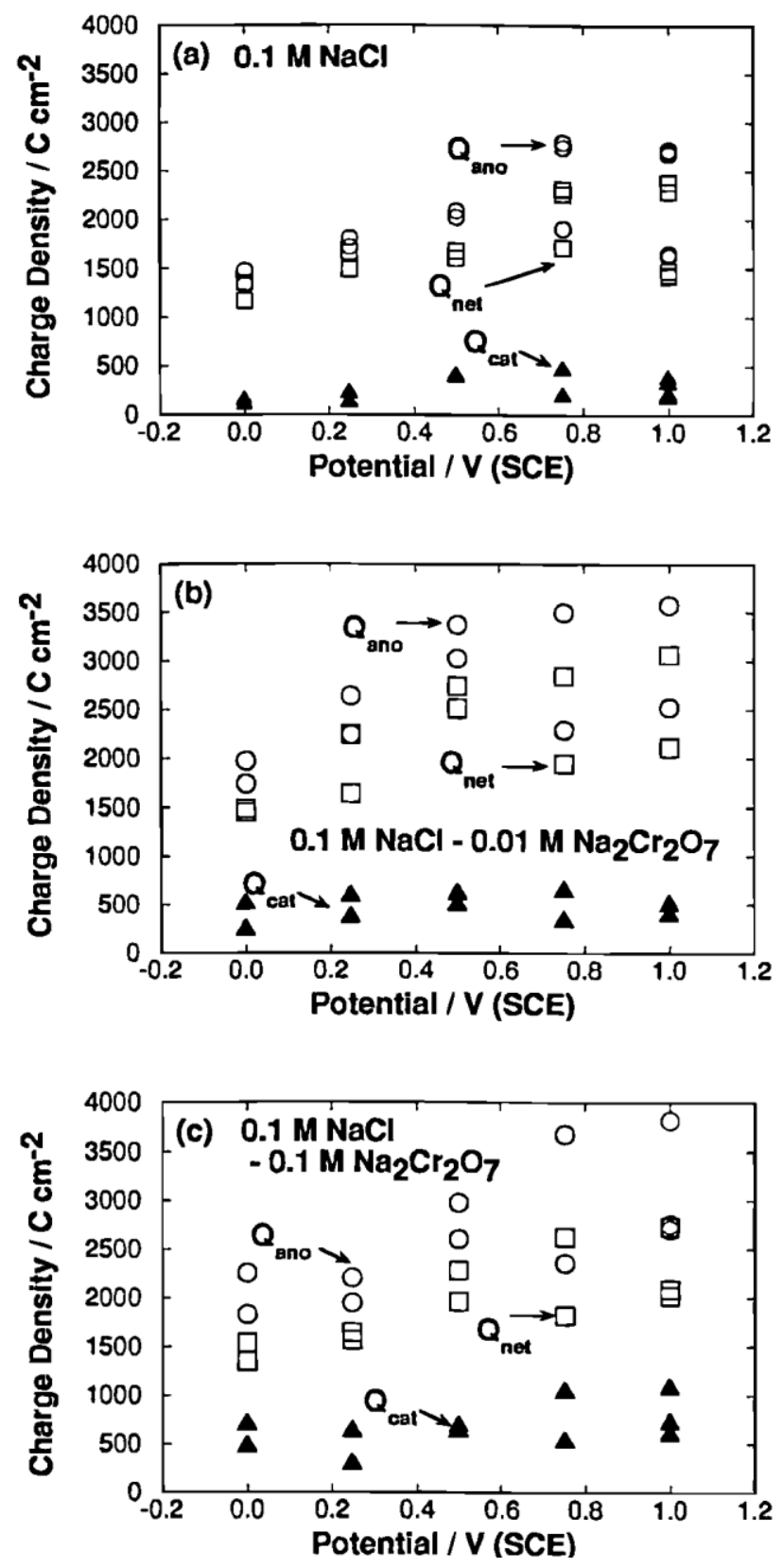

Figure 2. Effect of applied potential on $Q_{\text {ano }}, Q_{\text {net }}$, and $Q_{\text {cat }}$ for different dichromate concentrations: (a) $0.1 \mathrm{M} \mathrm{NaCl}$, (b) $0.1 \mathrm{M} \mathrm{NaCl}-0.01 \mathrm{M} \mathrm{Na}_{2} \mathrm{Cr}_{2} \mathrm{O}_{7}$, (c) $0.1 \mathrm{M} \mathrm{NaCl}-0.1 \mathrm{M} \mathrm{Na}_{2} \mathrm{Cr}_{2} \mathrm{O}_{7}$.

It should be noted that the observations in this work are limited to the specific situation of dissolution kinetics in pits and crevices. The environment in pits and crevices is likely to be rather acidic, so that the concentration of doubly charged chromate anions, $\mathrm{CrO}_{4}^{2-}$, would be very low. The $\mathrm{pH}$ for the different bulk solutions with different dichromate concentrations varied from about 4.3 to about 6.1. This variation was considered to be insignificant relative to the acidification that occurs in the crevice environment. Forthcoming papers will describe the behavior of artificial crevice electrodes in chromate solutions having alkaline bulk pH values. 
The minimal effect of a large dichromate ion concentration on crevice dissolution kinetics might be explained by the absence in the acidic environment of chromate ions, which could be the active inhibiting species. This still indicates that corrosion inhibition of $\mathrm{Al}$ by $\mathrm{Cr}(\mathrm{VI})$ species is not the result of a reduction in the rate of active dissolution in a pit or crevice environment.

The addition of dichromate ions does increase the local cathodic reaction on Al slightly. The anodic and net charges tend to increase with applied potential. However, the data show scatter owing to poor reproducibility. The largest charges at each potential seem to increase linearly with applied potential, suggesting ohmic control. However, the smallest charges at each potential are almost independent of potential, suggesting diffusion control. The poor reproducibility may be a result of the complicated and irreproducible nature of hydrogen evolution in the crevice cells.

Figure 3 shows the net charge of dissolution for the initial 15 min of polarization for each solution. The image analysis for $Q_{\text {ano }}$ was performed only at the end of the $2 \mathrm{~h}$ experiment, so only the net charge is available for shorter times. The 15 min data are less scattered compared to the $2 \mathrm{~h}$ data, and clearly increase with applied potential. It is obvious that the dissolution is ohmic controlled, at least for shallow crevices. In Fig. 3, it is clear that the net charge is highest in the solution with highest concentration of dichromate. This is further support for the statement that dichromate ions do not act as an anodic inhibitor under the condition of potentiostatically controlled active pitting or crevice corrosion.

The influence of dichromate concentration on various charges can best be viewed by considering charge ratios, Fig. 4, because the charges are functions of applied potential. The dichromate reduction reaction increases with dichromate concentration. It accounts for about one-third of the total cathodic reaction and is equal to about $9 \%$ of the anodic charge when present at a concentration of $0.1 \mathrm{M}$. Furthermore, $Q_{\text {cat }} / Q_{\text {ano }}$ increases from about $15 \%$ to about $26 \%$ as the

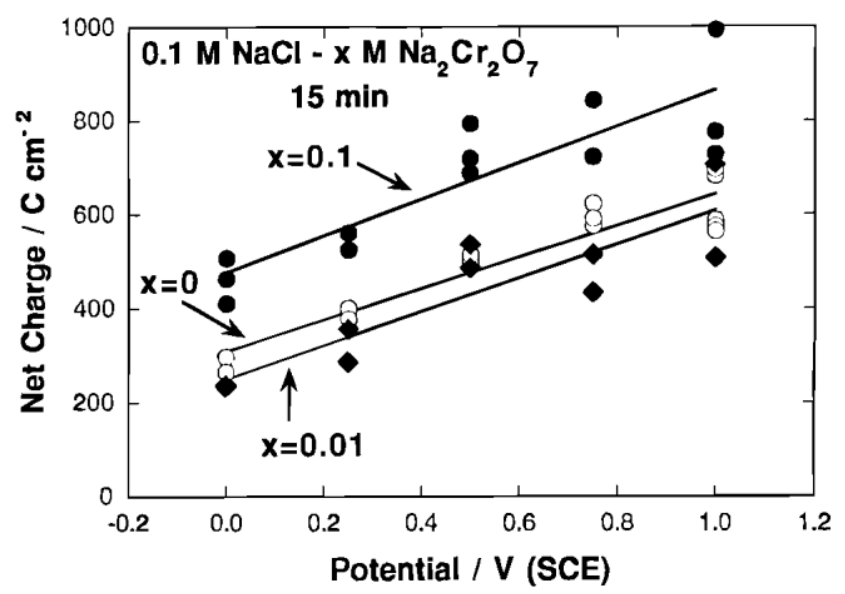

Figure 3. Effect of applied potential on the net charge of initial $15 \mathrm{~min}$ of polarization.

dichromate concentration increases to $0.1 \mathrm{M}$. Interestingly, the dichromate reduction taking place at the electrode surface apparently does not significantly reduce the anodic dissolution kinetics. The charge associated with hydrogen evolution is almost independent of dichromate 
concentration. Accordingly, the charge associated with the cathodic reactions is increased by the reduction of dichromate ions. The ratio $Q_{\mathrm{H} 2} / Q_{\mathrm{ano}}$ obtained in the present work, about $15 \%$, is similar to that found for $\mathrm{Al}$ artificial pit electrodes in saturated $\mathrm{AlCl}_{3}{ }^{4}$ and for $2 \mathrm{D}$ pits in $\mathrm{Al}$ thin films growing in dilute chloride solutions. ${ }^{14}$

Under pure ohmic control, the net current density is given by the ratio of the voltage drop to the resistance

$$
i_{\text {net }}=\Delta E / A R=\Delta E / R_{\mathrm{s}} \delta
$$

where $i_{\text {net }}\left(\mathrm{A} / \mathrm{cm}^{2}\right)$ is the net current density, $\Delta E(\mathrm{~V})$ is the potential difference that is creating the ohmic potential drop, $A\left(\mathrm{~cm}^{2}\right)$ is the active electrode area, $R(\Omega)$ is the average solution resistance in the crevice, $R_{\mathrm{S}}(\Omega \mathrm{cm})$ is the average specific resistance of the electrolyte in the crevice, and $\delta$ $(\mathrm{cm})$ is the depth of the crevice. The net current density must be considered instead of total anodic current density because the ohmic potential drop results only from the net current flowing out of the crevice. The ratio $Q_{\text {cat }} / Q_{\text {ano }}=R_{\text {ca }}$ is shown in Fig. 4 to vary from about 0.15 to 0.26 as the dichromate concentration increases from 0 to $0.1 \mathrm{M}$. The ratio of the local cathodic current density to the anodic current density is assumed to be equal to $R_{\text {ca }}$, so $i_{\text {net }}=\left(1-R_{\mathrm{ca}}\right) i_{\text {ano. }}$. The total anodic current density can be defined by Faraday's law

$$
i_{\text {net }}=\left(1-R_{\mathrm{ca}}\right) i_{\mathrm{ano}}=\left(1-R_{\mathrm{ca}}\right)(d \delta / d t)(\rho n F / M)
$$

where $t(\mathrm{~s})$ is time. Rearranging Eq. 3 and 4 gives

$$
\delta d \delta=\Delta E M d t /\left(1-R_{\mathrm{ca}}\right) n F R_{\mathrm{s}} \rho
$$

Integration of Eq. 5 yields

$$
\delta^{2}=2 \Delta E M t /\left(1-R_{\mathrm{ca}}\right) n F R_{\mathrm{s}} \rho
$$

Substituting Eq. 6 back into 3

$$
i_{\text {net }}=\Delta E / R_{\mathrm{s}} \delta=\left[\left(1-R_{\mathrm{ca}}\right) \rho n F \Delta E / 2 R_{\mathrm{s}} M\right]^{1 / 2} t^{-1 / 2}
$$

Rearranging yields 
The value of $1 / i_{\text {net }}^{2}$ as a function of time is shown in Fig. 5. Figure $5 b$ is the magnification of the area in the dotted square in Fig. 5a. In the initial stage of polarization, $1 / i^{2}$ is linearly related to polarization time, in accordance with ohmic control. After $20 \mathrm{~min}$ or more, there is a transition to a steeper slope, and then later there is another transition where the slope decreases. Figure 5 shows the results of two replications of the same experiment. The exact times for these

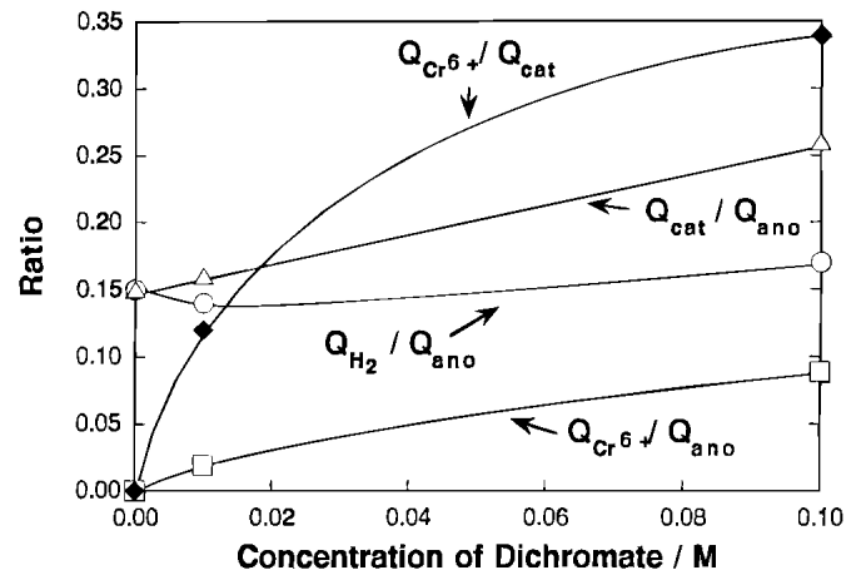

Figure 4. Effect of dichromate concentration on various charge ratios, $\mathrm{Q}_{\mathrm{Cr}} 6+/ \mathrm{Q}_{\text {cat }}, \mathrm{Q}_{\text {cat }} / \mathrm{Q}_{\text {ano }}, \mathrm{Q}_{\mathrm{H} 2} / \mathrm{Q}_{\text {ano }}$, and $\mathrm{Q}_{\mathrm{Cr}} 6+/ \mathrm{Q}_{\mathrm{ano}}$. 


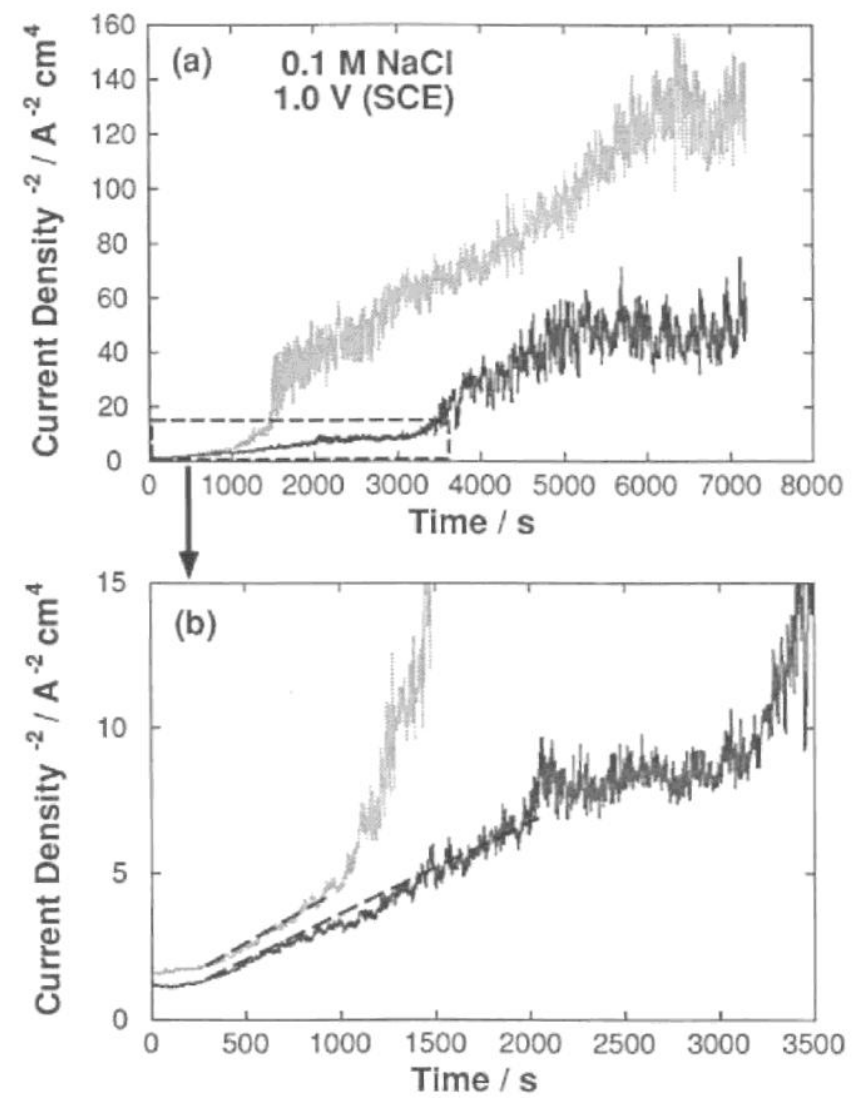

Figure 5. The change in the value of $1 / i^{2}$ with time during the potentiostatic polarization for two different experiments at $1.0 \mathrm{~V}$ (SCE) in $0.1 \mathrm{M} \mathrm{NaCl}$ solution.

transitions are not reproducible, which was typical for all conditions, and is likely the reason for the irreproducibility of the charges as seen in Fig. 2. All of the data exhibited the first transition and about $75 \%$ of the data exhibited the second transition.

Since conductivity is the inverse of resistivity, Eq. 8 can be rearranged to give

$$
i_{\text {net }}^{2} t=\left(1-R_{\mathrm{ca}}\right) \rho n F \Delta E / 2 R_{\mathrm{s}} M=\left(1-R_{\mathrm{ca}}\right) \rho n F \sigma\left(E_{\mathrm{app}}-E_{\mathrm{bot}}\right) / 2 M[9]
$$

where $\sigma\left(\Omega^{-1} \mathrm{~cm}^{-1}\right)$ is the average conductivity inside of the crevice, $E_{\text {app }}$ is the applied potential relative to the reference electrode in the bulk solution, and $E_{\mathrm{bot}}$ is the potential at the bottom of the artificial crevice electrode. The values of $i_{\text {net }}^{2} t$ for the initial stage of dissolution (the reciprocal of the slope in Fig. 5b) are plotted in Fig. 6 for each solution as a function of applied potential. By fitting lines to the data in Fig. 6, and applying Eq. 9, it is possible to extract values for the crevice conductivity, $\sigma$, and the potential at the bottom of crevice, $E_{\text {bot. }}$ In this analysis it is assumed that both of these parameters, $\sigma$ and $E_{\mathrm{bot}}$, are constant with applied polarization potential. Interestingly, $\sigma$ and $E_{\mathrm{bot}}$ are not monotonic functions of dichromate concentration. The conductivity of the crevice increases when $0.01 \mathrm{M} \mathrm{Na}_{2} \mathrm{Cr}_{2} \mathrm{O}_{7}$ is added to $0.1 \mathrm{M} \mathrm{NaCl}$ as might be expected from the added ionic strength. The conductivities of bulk solutions of $0.1 \mathrm{M} \mathrm{NaCl}, 0.1$ 
$\mathrm{M} \mathrm{NaCl}+0.01 \mathrm{M} \mathrm{Na}_{2} \mathrm{Cr}_{2} \mathrm{O}_{7}$, and $0.1 \mathrm{M} \mathrm{NaCl}+0.1 \mathrm{M} \mathrm{Na}_{2} \mathrm{Cr}_{2} \mathrm{O}_{7}$ are $10.0,11.5$, and $24.7 \mathrm{mS} / \mathrm{cm}$, respectively. However, the conductivity of the crevice decreases to a value lower than that of 0.1 $\mathrm{M} \mathrm{NaCl}$ when $0.1 \mathrm{M} \mathrm{Na}_{2} \mathrm{Cr}_{2} \mathrm{O}_{7}$ is added.

The estimated potential at the bottom of the crevice is shown in Fig. 7 as a function of the estimated conductivity inside of the crevice. It is shown that the bottom potential decreased as the crevice conductivity decreased, which is reasonable since the bottom potential will decrease

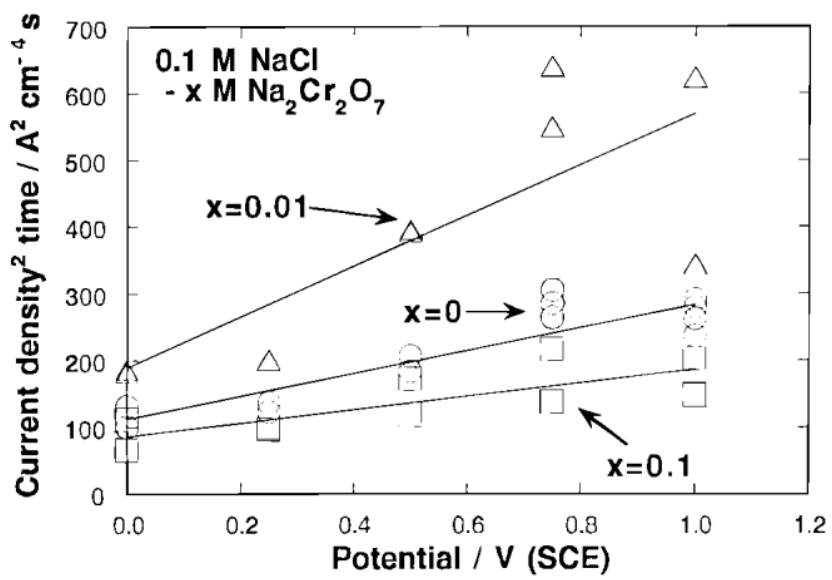

Figure 6. The influence of applied potential on $i^{2} t$ in the ohmic portion of the current transients.

with increasing ohmic potential drop. Apparently hydrogen evolution is again controlling the process. Estimated conductivity of the crevice and the bottom potential are shown as a function of $Q_{\mathrm{H} 2} / Q_{\text {ano }}$ in Fig. 8, and a monotonic relation is observed. The conductivity of the crevice decreased when $Q_{\mathrm{H} 2} / Q_{\text {ano }}$ increased because of the insulating nature of hydrogen bubbles; no direct influence of the concentration of dichromate ions on the conductivity or the bottom potential is seen. The conductivity in the crevice and thus the dissolution role seems to be controlled by hydrogen evolution, and indirectly by dichromate concentration.

The conductivity of the solution in the crevice estimated during the initial ohmically control process decreased with increasing of the ratio of the charge of hydrogen evolution, $Q_{\mathrm{H} 2}$, to the anodic charge, $Q_{\text {ano }}$ as shown in Fig. 8. Although the conductivity of the bulk solution increases simply with concentration of dichromate ions, the conductivity inside of the crevice is not directly related with dichromate concentration in bulk solutions. The hydrogen bubbles can be responsible for the decrease in the conductivity inside of the crevice. It is assumed that the nature of evolved hydrogen gas in the crevices has a large influence on the mass transport and also controls the conductivity. The motion of the hydrogen bubbles in the crevice will cause considerable mixing in the crevice and will prevent the increase in ion concentration and resultant increase in conductivity. The presence of hydrogen bubbles in the crevice furthermore will increase the ohmic resistance because the hydrogen bubble itself is an insulator. The conductivity of the crevice in a solution with $0.1 \mathrm{M} \mathrm{NaCl}+0.1 \mathrm{M} \mathrm{Na}_{2} \mathrm{Cr}_{2} \mathrm{O}_{7}$ is the lowest despite the fact that the conductivity of the bulk solution is the highest. The amount of hydrogen 


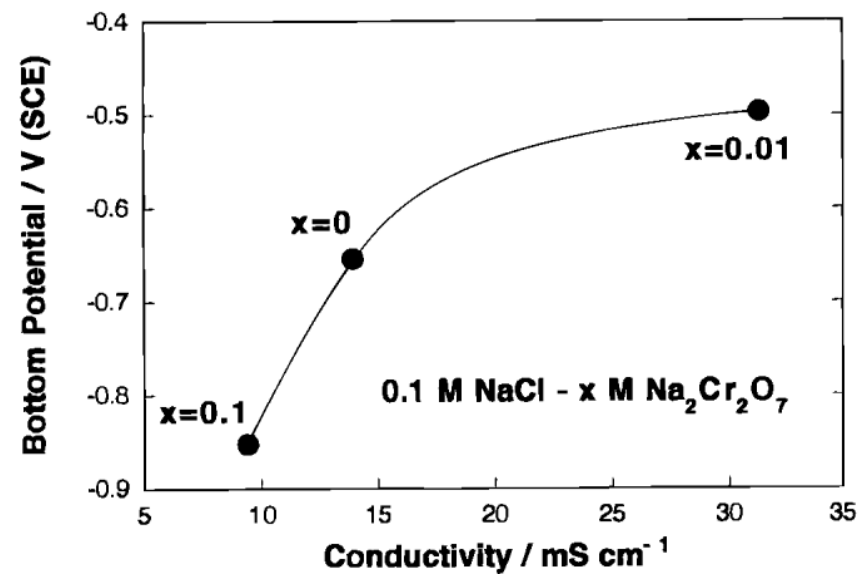

Figure 7. The relation between the estimated conductivity and bottom potential in $0.1 \mathrm{M} \mathrm{NaCl}-x \mathrm{M} \mathrm{Na} \mathrm{Cr}_{2} \mathrm{O}_{7}$.

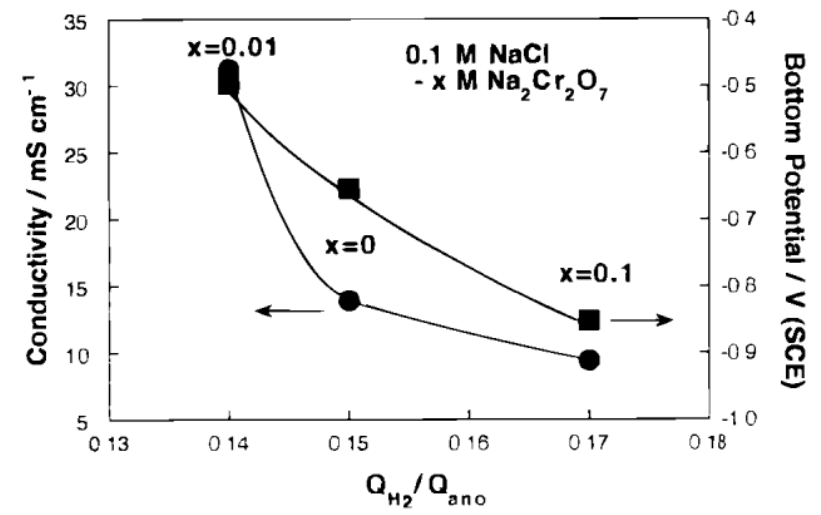

Figure 8. The influence of the ratio of hydrogen evolution charge to anodic dissolution charge on conductivity and bottom potential of the crevice.

gas evolved in the solution containing $0.1 \mathrm{M} \mathrm{Na}_{2} \mathrm{Cr}_{2} \mathrm{O}_{7}$ is the highest because the charge in the initial stage of dissolution and $Q_{\mathrm{H} 2} / Q_{\text {ano }}$ in the solution are the highest as seen in Fig. 3 and 4. Even though dissolved aluminum ions may increase the conductivity of the solution inside of the crevice, the convection by hydrogen bubbles prevents the concentration of ions. Thus, it can be assumed that the hydrogen evolution in the solution with $0.1 \mathrm{M} \mathrm{Na}_{2} \mathrm{Cr}_{2} \mathrm{O}_{7}$ resulted in the lowest conductivity. The effect of dichromate ions on the dissolution kinetics is not direct but it may affect the condition of the crevice by influencing hydrogen evolution.

The initial net dissolution charge increases with polarization potential as shown in Fig. 3 . This fact clearly indicates that the dissolution is ohmic or mixed ohmic/charge transfer controlled.

After the initial stage of dissolution, the value of $1 / i^{2}$ increased linearly with time, showing a different slope than that of the initial dissolution stage. There are several possible explanations for the change in behavior with increasing crevice depth. If the increase in the concentration of corrosion products inside of the crevice leads to saturation of the metal chloride 
at the bottom of the crevice, the dissolution kinetics would change to diffusion control. Under diffusion control, the dissolution current density is described as follows assuming that the diffusion layer thickness is equal to the crevice depth, $\delta$

$$
i_{\text {ano }}=n F D^{\prime}\left(C_{\mathrm{s}}-C_{\mathrm{b}}\right) / \delta=(d \delta / d t)(\rho n F / M)
$$

where $D^{\prime}\left(\mathrm{cm}^{2} \mathrm{~s}\right)$ is the diffusion coefficient corrected for migration. $C_{\mathrm{b}}$ and $C_{\mathrm{s}}\left(\mathrm{mol} / \mathrm{cm}^{3}\right)$ are the bulk and saturation concentration of the metal ions, respectively.

Integration of Eq. 10 yields

$$
\delta^{2}=2 M D^{\prime}\left(C_{\mathrm{s}}-C_{\mathrm{b}}\right) t / \rho
$$

Substituting into Eq. 10

$$
1 / i_{\text {ano }}^{2}=2 M t /\left[(n F)^{2} D^{\prime}\left(C_{\mathrm{s}}-C_{\mathrm{b}}\right) \rho\right]
$$

These equations describing diffusion-controlled dissolution are analogous to those for the ohmiccontrolled case (Eq. 3-9) where the reciprocal of the current density for the diffusion control process, $1 / i_{\text {ano }}^{2}$, is also proportional to time. So the linear dependence of $1 / i_{\text {ano }}^{2}$ on time at longer times (with lower slope) is also consistent with diffusion control.

The time of transition from the initial ohmic-controlled dissolution to the second dissolution process showing different slope of $1 / i_{\text {ano }}^{2} v s$. time curve is not reproducible. This is probably because the nature of hydrogen evolution is complicated and irreproducible. The rate of local hydrogen evolution on the aluminum electrode decreases with decreasing anodic dissolution current. When the hydrogen evolution is vigorous, the solution inside of the crevice is stirred considerably, and the buildup of metal ion concentration or corrosion products cannot occur. As the depth of the crevice increases, the dissolution current decreases accompanied by decreasing hydrogen evolution. Though the ratio of local cathodic current to anodic dissolution current may not change, the efficiency of mixing of the solution inside of the crevice and preventing the concentration of ions is lowered with the decrease in the hydrogen evolution. Thus, eventually the concentration of the corrosion product at the bottom of crevice may reach saturation.

Diffusion-controlled dissolution kinetics of artificial pit electrode for stainless steel, ${ }^{1,3}$ iron, ${ }^{5,8}$ and titanium ${ }^{8}$ has been reported. In the case of aluminum, however, it is reported that dissolution is ohmic controlled because of agitation of electrolyte in the crevice by hydrogen bubbles generated in the pits. ${ }^{8,15}$ In contrast, others have reported that diffusion-controlled dissolution of aluminum can occur. Wong and Alkire ${ }^{16}$ initiated single pits by an ion implantation technique, and studied their growth under potentiostatic control in $1 \mathrm{M} \mathrm{NaCl}, \mathrm{pH}$ 11 , at $-0.5 \mathrm{~V}(\mathrm{SCE})$. They indicated that the current density was in agreement with theoretical 
predictions based on diffusion-controlled dissolution of a precipitated aluminum oxychloride film. Similar results were found for laser-initiated single pits in pure aluminum. ${ }^{17,18}$ Beck has reported that a combination of concentrated aluminum chloride solution (more than $80 \%$ of saturation) and high anodic potentials will result in formation of a continuous salt film on aluminum. ${ }^{4}$ Although the condition of the solution and applied potential of the present study is less suitable for salt film formation than that Beck used, the size of the artificial crevice of this study, $0.0508 \times 6.7 \mathrm{~mm}$ is smaller than Beck's, $1.6 \times 3.2 \mathrm{~mm}$. It is possible that the smaller size of the crevice decreases the efficiency of transportation of corrosion products from the artificial crevice to the bulk solution and helps saturation of corrosion product.

As the depth of the crevice increases, the likelihood of bubbles getting stuck in the narrow slot also increases. Hydrogen bubbles stuck in the crevice would be effective barriers to both current flow and mass transport. Stuck bubbles may therefore increase the tendency for salt film precipitation. It is also possible, however, that the decrease of the slope of the $1 / i^{2} v s$. time curve is caused by a decrease in the conductivity of the crevice. Because a hydrogen bubble itself is an insulator, hydrogen bubbles stuck in the crevice may control the conductivity of the crevice, thereby decreasing the $1 / i^{2} v$ s. $t$ slope.

Finally, it is possible that partial precipitation of corrosion product increases the resistance in the crevices. Even if the formation of a salt film layer covering the entire aluminum surface may be difficult because of hydrogen evolution, partial precipitation of corrosion product can take place when the hydrogen evolution is not vigorous and the efficiency of stirring the electrolyte is not sufficient.

Although the dissolution mechanism in the later stage of crevice growth is not quite clear, it is thought that hydrogen evolution controls the dissolution mechanism and the time of the transition from the initial stage of dissolution. It can be said that the irreproducible nature of hydrogen bubbles is responsible for the poor reproducibility of the data in Fig. 2. The edges of the aluminum electrodes were uneven after the polarization, especially when the depth of the crevice was deep. The nonflat edge of the aluminum electrode must be caused by hydrogen evolution. The nonuniform distribution of depth of crevice may also complicate the dissolution mechanism.

While the evolution of hydrogen during these experiments resulted in poor reproducibility and complications in interpretation, hydrogen evolution is a real part of the Al localized corrosion process. The phenomena observed in these experiments can occur in actual Al pits and crevices.

\section{Conclusions}

The dissolution kinetics of aluminum in a crevice in chloride-containing solutions and the effect of dichromate ions on the crevice growth were investigated by the artificial crevice electrode technique. The addition of dichromate ions did not suppress the active crevice dissolution. Therefore, the mechanism of localized corrosion inhibition by chromates must be something other than anodic inhibition in the pit or crevice environment. The charge of local cathodic reactions on aluminum electrode increased with addition of dichromate because the reduction reaction of dichromate took place on the electrode.

The initial dissolution in a crevice showed ohmic control. Under ohmic control, the addition of dichromate ions changed the conductivity of the crevice and the potential at the bottom. The conductivity in the crevice and thus the dissolution rate seems to be controlled by 
hydrogen evolution, and only indirectly by dichromate concentration.

\section{Acknowledgments}

This work was supported by the Air Force Office of Scientific Research under contract no. F49620-96-10479. H. Isaacs, S. Smialowska, and R. Buchheit are gratefully acknowledged for interesting discussions.

The Ohio State University assisted in meeting the publication costs of this article.

\section{References}

1. J. W. Tester and H. S. Isaacs, J. Electrochem. Soc., 122, 1438 (1975).

2. R. C. Newman and H. S. Isaacs, J. Electrochem. Soc., 130, 1621 (1983).

3. H. S. Isaacs, J. Electrochem. Soc., 120, 1456 (1973).

4. R. T. Beck, Electrochim. Acta, 29, 485 (1984).

5. R. T. Beck, J. Electrochem. Soc., 129, 2412 (1982).

6. H. S. Isaacs and R. C. Newman, in Corrosion Chemistry within Pits, Crevices and Cracks, A. Thurnbull, Editor, p. 45, HMSO Publications, London (1987).

7. P. Heimgartner and H. Bohni, Corrosion, 41, 715 (1985).

8. T. R. Beck, Corrosion, 33, 9 (1977).

9. H. S. Isaacs, J.-H. Cho, M. L. Rivers, and S. R. Sutton, J. Electrochem. Soc., 142, 1111 (1995).

10. G. S. Frankel, A. J. Davenport, H. S. Isaacs, A. G. Schrott, C. V. Jahnes, and M. A. Russak, J. Electrochem. Soc., 139, 1812 (1992).

11. G. S. Frankel, A. G. Schrott, A. J. Davenport, H. S. Isaacs, C. V. Johnes, and M. A. Russak, J. Electrochem. Soc., 141, 83 (1994).

12. U. Steinsmo and H. S. Isaacs, J. Electrochem. Soc., 140, 643 (1993).

13. R. C. Newman, Corros. Sci., 25, 341 (1985).

14. G. S. Frankel, Corros. Sci., 30, 1203 (1990).

15. F. Hunkeler and H. Bohni, Corrosion, 40, 534 (1984).

16. K. P. Wong and R. C. Alkire, J. Electrochem. Soc., 137, 3010 (1990).

17. R. Alkire and M. Feldman, J. Electrochem. Soc., 135, 1850 (1988).

18. D. W. Buzza and R. C. Alkire, J. Electrochem. Soc., 142, 1104 (1995). 